\title{
The effect of additional staple in the tibial fixation of anterior cruciate ligament reconstruction: a biomechanical study in sheep tibia
}

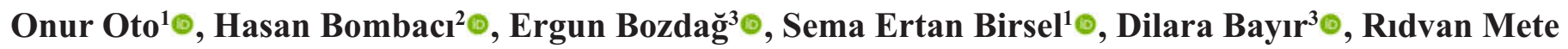 \\ Oral $^{2} \odot$
}

${ }^{1}$ Department of Orthopedics and Traumatology, Istanbul Başakşehir Çam and Sakura City Training and Research Hospital, Istanbul, Turkey; ' ${ }^{2}$ Department of Orthopedics and Traumatology, University of Health Sciences, Haydarpaşa Numune Training and Research Hospital, Istanbul, Turkey; ${ }^{3}$ Laboratory of Strength of Materials and Biomechanics, Istanbul Technical University, Faculty of Mechanical Engineering, İstanbul, Turkey

\section{ABSTRACT}

Objectives: The aim of this study was to evaluate the necessity of staple use and the importance of screw diameter in the anterior cruciate ligament (ACL) reconstruction.

Methods: Twenty-eight sheep hind legs were divided into four groups in terms of tibial side fixation type as $7 \mathrm{~mm}$ bioabsorbable screw (Group 1), $7 \mathrm{~mm}$ bioabsorbable screw and staple (Group 2), $8 \mathrm{~mm}$ screw (Group 3 ), and $8 \mathrm{~mm}$ screw and staple (Group 4). The maximum force causing insufficiency in the graft, and the fixation strength between the groups were recorded. The results were compared among the groups.

Results: As a result of the statistical analysis regarding the necessity of using staple, group 2, with $7 \mathrm{~mm}$ screws and staple, was found to be significantly more resistant than group $1(p=0.018)$. In consequence of the biostatistics study, staple use was found not to create a statistically significant difference between group 3 in which an $8 \mathrm{~mm}$ bioabsorbable screw was used and group $4(p=0.805)$. In the statistical study to understand the significance of screw diameter, the samples of group 1 and group 3, in which no staple was used, were compared. It was found that group 3 samples with $8 \mathrm{~mm}$ screws had higher strength than those with $7 \mathrm{~mm}$ screws $(p=0.011)$. Between group 2 and group 4 in which a staple was used, despite the increase in screw diameter, the value of maximum strength had no remarkable difference statistically $(p=1.00)$.

Conclusions: Fixation strength is higher when fixation was done with a screw $1 \mathrm{~mm}$ larger than tibial tunnel diameter. However, using either a screw with $1 \mathrm{~mm}$ wider than tibial tunnel diameter and the screw in the same diameter with the tibial tunnel and additional staple have similar fixation strength on the tibial side. Therefore, we conclude that screw width is more important in maintaining stability of the graft fixation.

Keywords: ACL reconstruction, biomechanics, tibial fixation, bioabsorbable screw, staple

A nterior cruciate ligament (ACL) is the most frequently injured ligament in the knee, especially during sportive activities. Arthroscopic ACL is usually performed in patients who are at young ages. During
ACL reconstruction, the tibial side is the weakest point of the reconstruction [1-4]. There are two main reasons for that. Firstly, bone density of the proximal tibia is lower than the distal femur and secondly, force direc- 
tion on the graft is parallel to the tibial tunnel [5]. It has been shown by many studies that graft fixation, especially on the tibial side, is the key point for the graft to remain stable in the early physical therapy program $[4,6]$. Therefore, tibial fixation must be strong enough to withstand an early effective rehabilitation program. A significant number of studies have shown that long screws with large diameter provide stronger fixation [6-9]. However, the larger screws may cause damage in the autograft [10].

On the other hand, fixation of graft on the tibial side with a staple in addition to bioabsorbable screw is a frequently used method to prevent failure as much as possible. In addition to the staple, post screw or washer screw can be used for fixation. Nevertheless, the use of additional fixation material has complications such as the subcutaneous sensation of the implant and pain. Therefore, a second surgical intervention may be required [11]. The advantage of fixation with staple among them is that it remains almost at the same level as the bone after insertion. Therefore, the staple is usually preferred due to the possibility of the patient to feel the implant is greatly reduced.

There are many publications advocating that bioabsorbable screws and staple should be used, and many others are defending and opposing the idea that fixation should be done with screw $1 \mathrm{~mm}$ wider than the tunnel width. However, currently there is no consensus regarding optimal tibial fixation in ACL reconstruction. In the present study, by testing two variables (staple usage and screw diameter) together, we intended to examine the relationship between them and the importance of each in the tibial fixation of ACL autograft. We aimed to evaluate the necessity of staple as an additional fixation method and the importance of screw diameter in the anterior cruciate ligament (ACL) reconstruction. We hypothesized that additional staple fixation increases the stability of graft when a screw of the same diameter was used.

\section{METHODS}

In the study, two years old on average, showing no additional pathology and slaughtered not more than 24 hours before the test, 28 sheep hind legs were used. In this biomechanical study, the tests were carried out in the Strength and Biomechanics Laboratory of the
Mechanical Engineering Faculty of Istanbul Technical University. MTS 858 Mini Bionix 2 test machine (MTS System Corporation 14000 Technology Drive Eden Prairie, MN USA, 55344) was used for study. The study was conducted according to the Decleration of Helsinki. Ethical approval was obtained from the institutional review board (771/08/2018). The ideal of the study is to do it in a young human cadaver. However, sheep bone was preferred because of its easy accessibility, its weight close to human weight and its long bones that can be implanted. Taken on the morning of the experiment from an abattoir where animals are slaughtered daily, sheep hind legs cut in the last 24 hours were dissected (Fig. 1). Soft tissues were removed. The tibia and femur were separated. The tibia was cut $12 \mathrm{~cm}$ distal to the knee joint level (Fig. 2). In

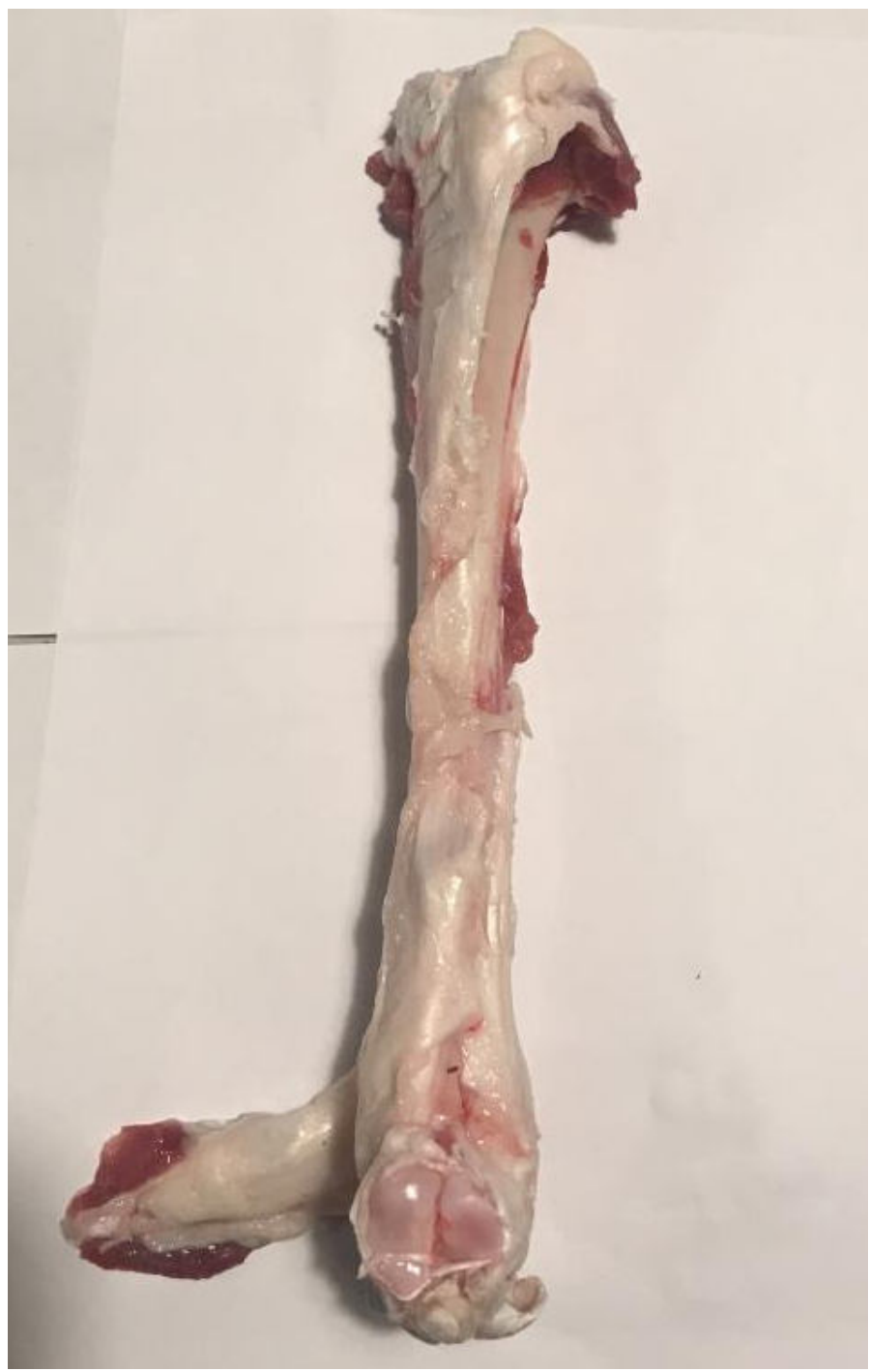

Fig. 1. Sheep tibia before fleksor digitorum tendon is removed. 


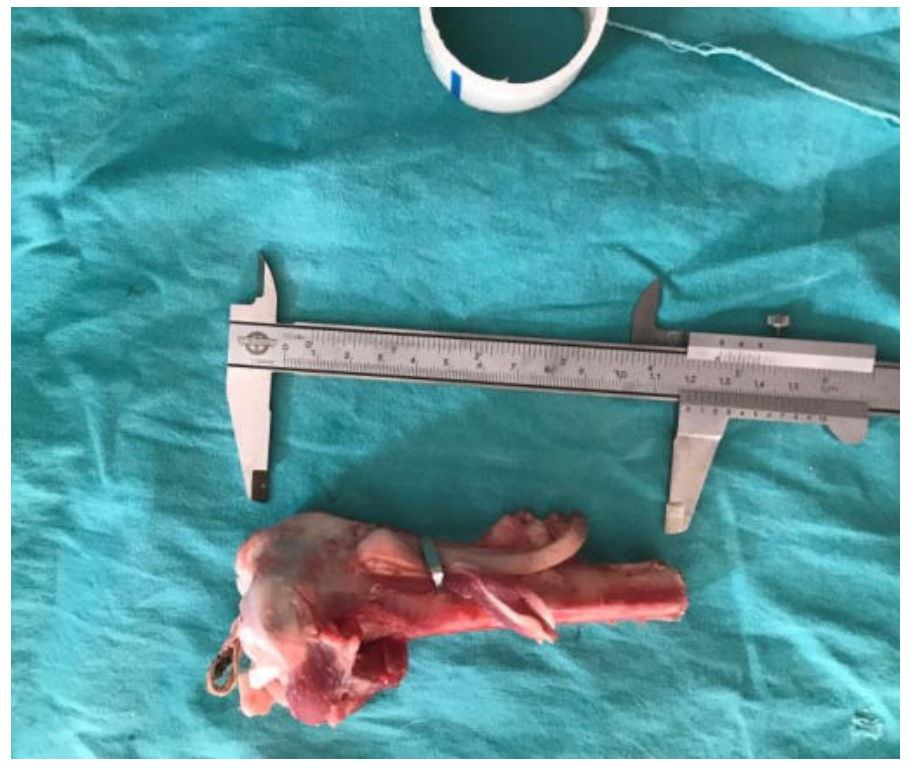

Fig. 2. Sheep tibia after flexor tendon graft is removed and cut below the knee joint level.

a previous study, the digitorum tendon was found to be an appropriate graft for ACL recontruction [12]. The flexor digitorum tendon was taken from the same leg of the same sheep as a graft (Fig. 3).

The grafts folded in half and were prepared to be $7 \mathrm{~mm}$ wide. With an average of $4 \mathrm{~cm}$, the tunnel was drilled with a $7 \mathrm{~mm}$ drill in the tibial joint in such a way that it would come out of ACL stump. The graft was passed through the tibial tunnel with $20 \mathrm{~mm}$ 'Continuous-Loop' (CL) (Arthtrex ${ }^{\circledR}$ ) and was inserted through the device to be tested via CL to prevent weak region formation in the graft (Fig. 4).

The samples were divided into four separate groups. In Group 1, only $7 \mathrm{~mm}$ wide bioabsorbable screws ( Tulpar biointerference screws ${ }^{\circledR}$ ) were used for tibial fixation. In Group 2, the graft was fixed to the tibial side with a $7 \mathrm{~mm}$ wide bioabsorbable screw and staple (Tulpar ligament staple ${ }^{\circledR}$ ). While $8 \mathrm{~mm}$ wide bioabsorbable screws were used in Group 3, $8 \mathrm{~mm}$ wide bioabsorbable screws and staple were used in Group 4.

To increase the involvement of the apparatus to which the tibia is fixed distally, two cross- $\mathrm{K}$ wires were sent and fixed to the apparatus to be tested with synthetic paste. CL was placed on the device with the help of a proximal steel hanger (MTS 858 Mini Bionix 2 ). The force was applied to compare fixation methods in the same direction as the tibial tunnel, the most challenging situation possible [13]. Thus, it was aimed to prevent the tension due to the angulation between the graft and the articular surface. Firstly, with a frequency of $2 \mathrm{~Hz}$, and a maximum of $50 \mathrm{~N}, 100$ cycle force was applied to the samples with force control, and then the

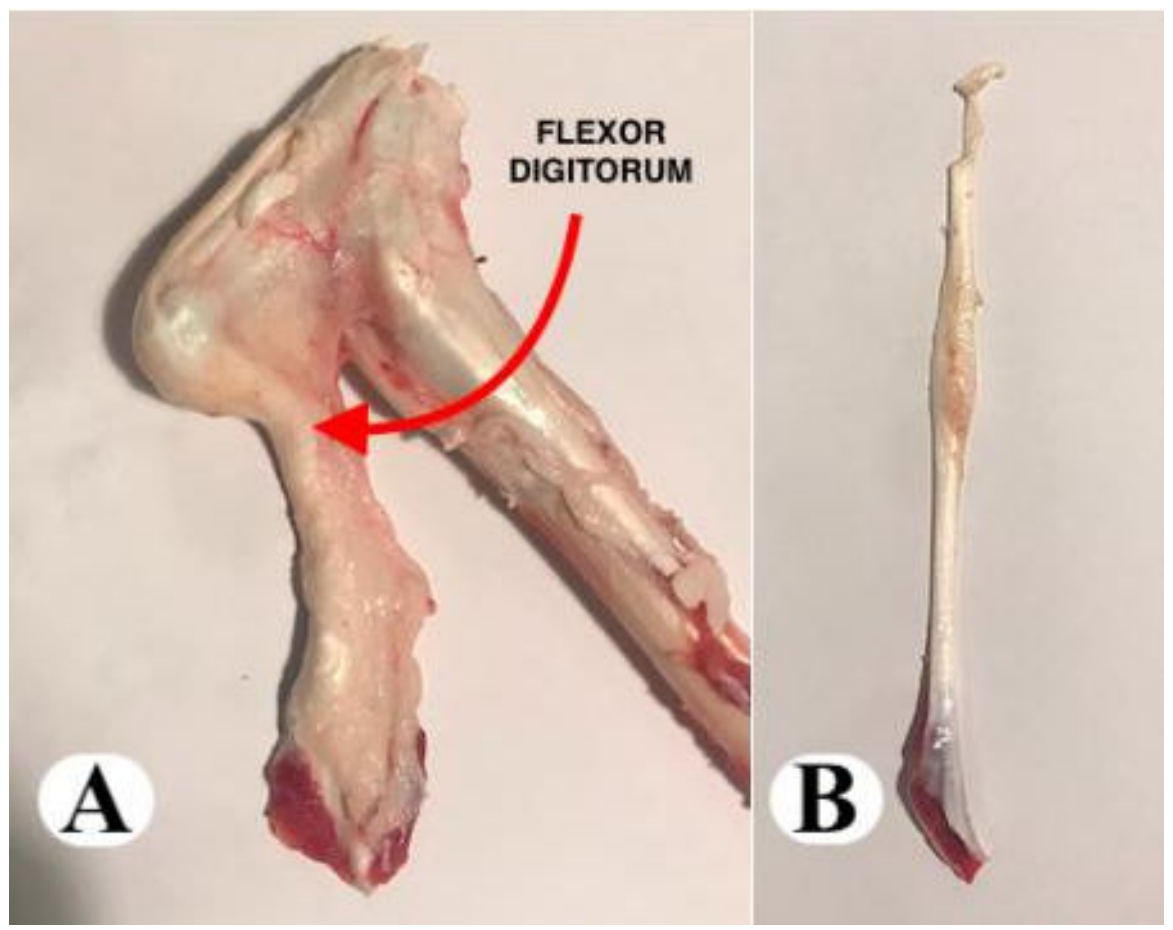

Fig. 3. (A) Hind legs flexor digitorum tendon of a 2-year old sheep, (B) Flexor digitorum tendon, which was used as a graft, after dissection. 


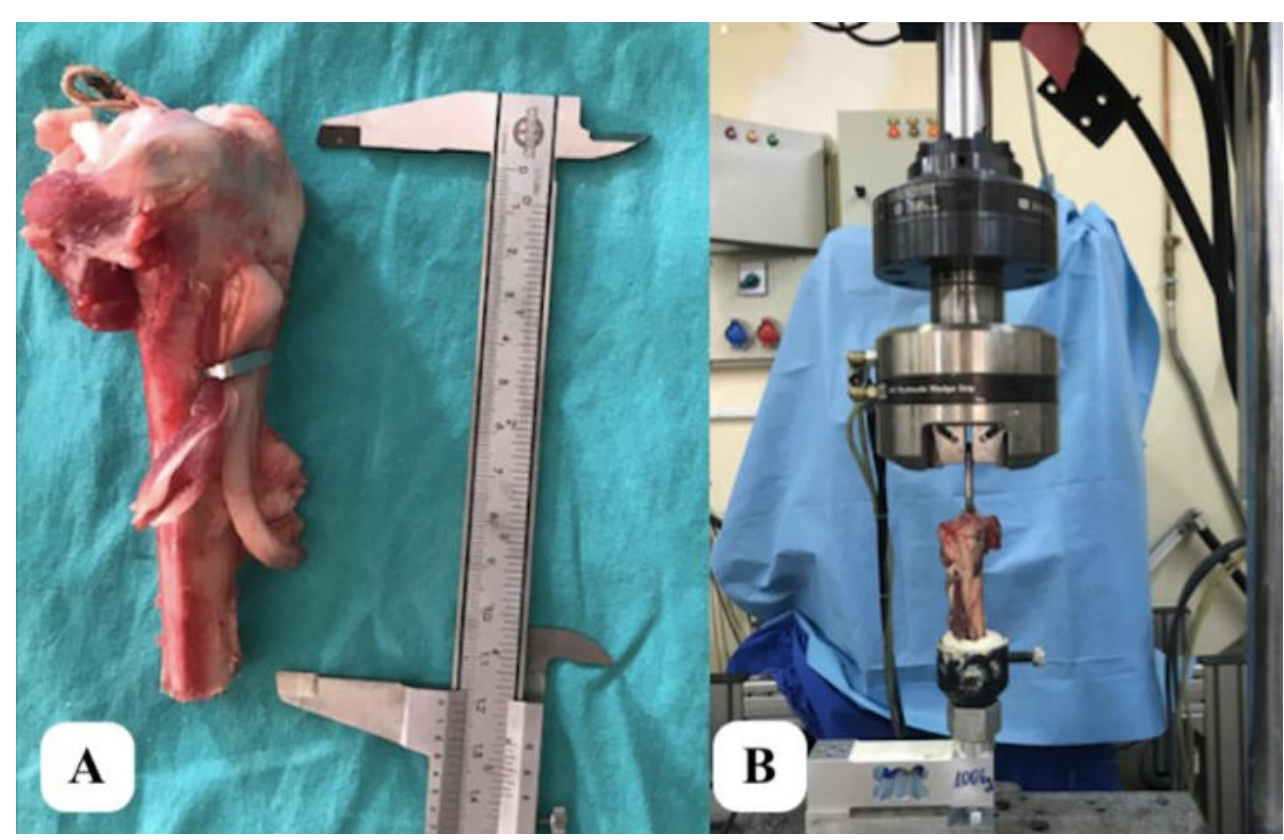

Fig. 4. (A) A $8 \mathrm{~mm}$ screw and staple used in the sample after cut which is located $12 \mathrm{~cm}$ distal to the knee joint level, (B) The study setup with MTS 858 Mini Bionix 2 tester.

system was discharged up to $10 \mathrm{~N}$. Following this stage, the load-to-failure test was started. After this stage, the load-to-failure test was started by switching the force-controlled protocol to displacement-controlled protocol and traction was applied at a speed of $20 \mathrm{~mm} / \mathrm{min}$ until the failure occurred. Force displacement values were recorded with MTS Axial Load cell $(10000 \mathrm{~N} / 100 \mathrm{Nm})$. Throughout the test, the values of motion (displacement), strength (stiffness), and ultimate failure load were recorded.

\section{Statistical Analysis}

All analyses were performed using SPSS version 22.0 software. Quantitative data were expressed as the mean \pm standard deviation (SD). The values of motion (displacement), strength (stiffness), and ultimate failure load displayed nonparametric distribution according to the Kolmogorov-Smirnov test. Mann-Whitney $\mathrm{U}$ test was used to assess the between the groups.

\section{RESULTS}

In all samples used in the study, it was observed that the grafts were damaged at the screw bone junction on the tibial side, the weakest place in ACL reconstruction surgery [5]. Two samples damaged from the middle of the graft were excluded from the study.
Moreover, one sample that ruptured from the apparatus to which it was fixed for the study and one sample broken due to device error during the study were also excluded.

As a result of the statistical analysis, in terms of the necessity of using a staple, there was a significant difference between Group 1 and Group 2 - the former was fixated using a $7 \mathrm{~mm}$ screw and the latter with a $7 \mathrm{~mm}$ screw and staple - and Group 2 was observed to resist to an average of $398.8 \mathrm{~N}$ (Table 1). This value was found to be significant compared to the Group 1 samples that could resist to an average of $262.3 \mathrm{~N}$. Group 2 was recorded to be able to resist higher strength than Group $1(p=0.018)$. When we compared the values of Group 3 and Group 4, it was seen that additional staple use did not make a statistically significant difference between the two groups (Table 1) $(p=0.805)$.

When the data were analysed in terms of screw diameter, the results of Groups 1 and 3 were compared, in which no additional staple was used. It was statistically determined that the group samples using $8 \mathrm{~mm}$ screws had a significantly higher strength with an average of $398 \mathrm{~N}$ compared to the average force to which the $7 \mathrm{~mm}$ screw could resist $(262 \mathrm{~N})$ (Table 2) $(p=$ 0.011 ). Between the Groups 2 and 4 in which an additional staple was used, it was observed that the value of the maximum resistance did not have a statistically 
significant difference despite the increased screw diameter (Table 2) $(p=1.00)$.

\section{DISCUSSION}

The main findings of this study are that the fixation strength is higher when fixation was performed with a screw $1 \mathrm{~mm}$ larger than the diameter of the tibial tunnel. The fixation strengths are comparable in the samples, which fixation was made with a screw $1 \mathrm{~mm}$ wider than the tibial tunnel and the ones fixed with a staple in addition to the screw in the same diameter of the tibial tunnel.

In the study carried out by Micucci et al., fixation was respectively provided with $8,9,10$ and $11 \mathrm{~mm}$ screws in the $9 \mathrm{~mm}$ tunnel. Maximum strength was found highest in the screw of $11 \mathrm{~mm}$; however the difference was not statistically significant [6]. It has been observed that as the screw width decreases, the maximum strength declines as well. But, when the highest forces, which the grafts were withstanding are considered, all values were found to be higher than the force that the graft would withstand in daily life [6]. The authors also reported that graft motion was less when the screw diameter equal to the tunnel size used [6]. In the study of Suk et al. [9], with $8 \mathrm{~mm}, 9 \mathrm{~mm}, 10 \mathrm{~mm}, 11$ $\mathrm{mm}$ and $12 \mathrm{~mm}$ screws, the grafts were fixed to the tibial tunnels drilled with $8 \mathrm{~mm}$ drill. As a result of their study, the graft strength is observed to be $20 \%$ more in the $11 \mathrm{~mm}$ screw compared to the $8 \mathrm{~mm}$ screw. It also was reported that there appears no increase in graft fixation strength by increasing interference screw diameter beyond $3 \mathrm{~mm}$ of the tunnel diameter [9]. On the other hand, in the study of 37 pig femurs by Morris et al. [10], grafts were fixed to $8 \mathrm{~mm}$ wide femoral tunnels with $7 \mathrm{~mm}, 9 \mathrm{~mm}$ and $10 \mathrm{~mm}$ wide metal interference screws. As a result of thieir study, it was seen that all the tendons fixed with $9 \mathrm{~mm}$ wide screws were damaged at the entrance of the femoral tunnel and failed. In fixations with $8 \mathrm{~mm}$ screw, it also was determined that there was a risk of $58 \%$ graft slippage, $38 \%$ graft cut out. According to this study, it was stated that fixation with the same width as the tunnel width or $1 \mathrm{~mm}$ smaller screw would be appropriate [10]. Our findings are comparable with Micucci et al.'s [6] and Suk et al.'s [9] studies. We found that a screw $1 \mathrm{~mm}$ wider than the tunnel diameter provides sufficient fixation without the need for staple use.

In the study conducted by Kurosaka et al. [14], tibial fixation with only interference screw and that with only a staple were compared and it was concluded that fixation with an only bioabsorbable screw was stronger. Gerich et al. [15], who conducted a similar study, found that the maximum strength of the group that was fixated with the interference screw was between $506 \mathrm{~N}$ and $758 \mathrm{~N}$ and it was only $558 \mathrm{~N}$ in the patients who were provided with staple fixation. In the light of these studies, it was concluded that the interference screw alone could withstand the maximum force that the graft will be exposed to in early physical therapy $[14,15]$. In the present study, unlike the Gerich et al.'s [15] study, the specimens using both bioabsorbable screw and staples in addition to the screw were investigated at the same time. We found that when a screw of the same diameter as the tunnel was used, usage of an additional staple increases the strength of fixation.

In the animal model, Bauer et al. [16] did not show significant differences in the samples using interference screw and staple compared with fixation made with an interference screw alone. It might be depending on the samples used, because the swine knee, which used in Bauer et al.'s [16], has a stronger cancellous bone compared to the lamb, which is used in the present study. In their study on the knees of 15 pigs, Lee et al. [2] examined the difference among using bioabsorbable screws only, bioabsorbable screws with staple use and bioabsorbable screws with Push-lock screws. As a result of the study, they found that additional fixation implants such as staple and screw did not contribute to the structural durability and stiffness of the graft considerably in cyclic loads [2]. However, when they continued the test and the forces that would cause damage to the graft were measured, the samples using additional fixation materials were found to withstand higher forces and as a result of the study. They stated that the use of additional fixation material might be significant in patients with poor bone quality, revision cases, elderly ones and those with metabolic diseases that will affect the bone quality [2]. In the study performed by Hill et al. [17], female patients who underwent ACL reconstruction with hamstring graft were observed by dividing them into two groups as metal interference screw and metal in- 
terference screw and staple used in tibial fixation. In the study, they found that there was a remarkable difference between the two groups with the Lachman test and KT 2000 arthrometer (MEDmetric, San Diego, CA) in 2 years follow up time [17]. However, when this difference was evaluated with Lysholm score and IKDC (International Knee Documentation Committee assessment), no clinically meaningful difference was found. The authors suggested that staple use is suitable for patients with soft bone in whom the bioabsorbable screw cannot provide sufficient fixation. Also, in this study, they found an increased anterior knee pain due to staple use [17]. In their study on sheep knee, Fabriciani et al. [1] compared the metal interference screw used with bone block, absorbable interference screw and staple, metallic screw and washer screw. As a result of the comparison, bioabsorbable screw and staple duo were found as the fixation method that could resist the average highest force. Our findings support these findings [1, 17]. Our results showed that bioabsorbable screw with supplementary fixation with a staple, improve the structural strength for the ultimate tensile load on the tibial side.

In the study performed by Prado et al. [18] on the pig knee, fixation with bioabsorbable screw in addition to staple and that with only bioabsorbable screw were compared and no significant difference was found. In the study conducted by Teo et al. [19], 31 patients between the ages of 18 and 35 who only used bioabsorbable interference screws and 33 patients who used bioabsorbable interference screws and staple were compared. In the comparison made with the physical examination, arthrometer, IKDC form and Lysholm form 1 year after surgery, no significant difference was found between two groups [20]. The common findings in latter two studies [18, 19], in which they were not able to show any differences in terms of fixation strength on the tibial side, fixation was made with a screw larger than the tibial tunnel. It is well known that wider screw provides better fixation, however, in relation with the insertion angle of the screw used to fix the graft and the patient's bone quality, the graft is quite often damaged at the screw-tendon-bone junction and this is more likely with oversized screw $[1,8]$. Therefore, we also tested the specimens fixed with a screw in the same diameter as the tibial tunnel. In our study, we found that when graft fixation was provided with $7 \mathrm{~mm}$ bioabsorbable screw in the tunnel drilled with $7 \mathrm{~mm}$, staple use increased durability. Therefore, we speculate that the use of a staple in addition to the screw, will increase the reliability of fixation especially in patients with soft bone and fixed with a bioabsorbable screw in the same diameter with the tibial tunnel.

All-inside technique is a safe alternative in which no staple is used and it is gaining popularity in the current practice $[20,21]$. We agree with the need for additional fixation particularly in female patients with low bone density $[2,17]$.

\section{Limitations}

One of the limitations of the study is that it was made in the sheep knee and only the tibia was used. Whereas the ideal of the study is to do it in the young cadaver knee. Also, long-term results such as osteolysis could not be evaluated. For this, it is necessary to work with young cadaver and live animals. In addition, the same length bioabsorbable interference screws

$(28 \mathrm{~mm})$ were used in this study. Therefore, the effect of the screw length could not be evaluated. However, in this study, we used fresh sheep tendons and bones which was reported that the density of sheep bone is closer to that of young human bone compared to the old cadaver [22]. The animals were slaughtered within 24 hours and were not underwent the freezing process, which makes our study unique. The effect of screw width and staple use in fixation was evaluated with all possible combinations with four different groups.

\section{CONCLUSION}

We conclude that, graft fixation with interference screw $1 \mathrm{~mm}$ wider than the width of the tibial tunnel provides sufficient stability on the tibial side of specimens with adequate bone quality. As the relevance of clinical practice; by fixation of the graft only with screws, complications caused by staple can be eliminated and the cost of surgery can be reduced. However, in case, we can't afford the risk of injury to the graft with a larger screw, we can use a screw of the same diameter as the tunnel, then a staple should be kept ready during surgery to use if required. 


\section{Authors' Contribution}

Study Conception: OO; Study Design: OO, DB, EB; Supervision: HB; Funding: OO; Materials: OO; Data Collection and/or Processing: OO, RMO, DB; Statistical Analysis and/or Data Interpretation: OO, EB, SEB; Literature Review: OO; Manuscript Preparation: $\mathrm{OO}$ and Critical Review: $\mathrm{HB}, \mathrm{OO}$.

\section{Conflict of interest}

The authors disclosed no conflict of interest during the preparation or publication of this manuscript.

\section{Financing}

The authors disclosed that they did not receive any grant during conduction or writing of this study.

\section{REFERENCES}

1. Fabbriciani C, Mulas PD, Ziranu F, Deriu L, Zarelli D, Millano G. Mechanical analysis of fixation methods for anterior cruciate ligament reconstruction with a hamstring tendon graft. An experimental study in sheep knees. Knee 2005;12:135-8.

2. Lee JJ, Otarodifard K, Jun BJ, McGarry MH, Hatch GF, Lee TQ. Is supplementary fixation necessary in anterior cruciate ligament reconstructions? Am J Sports Med 2011;39:360-5.

3. Patkowski M, Królikowska A, Reichert P. Comparison of bioabsorbable interference screws composed of poly-l-lactic acid and hydroxyapatite (PLLA-HA) to WasherLoc tibial fixation in patients after anterior cruciate ligament reconstruction of the knee joint. Polim Med 2016;46:53-8.

4. Sun T, Zhang H. Biomechanical comparison of a novel tibial fixation technique versus interference screw fixation for ACL reconstruction using soft tissue grafts. Orthop J Sports Med 2021;9:23259671211028559.

5. Brand J Jr, Weiler A, Caborn DN. Graft Fixation in cruciate ligament reconstruction. Am J Sports Med 2000;28:761-74.

6. Micucci CJ, Frank DA, Kompel J, Muffly M, Demeo PJ, Altman GT. The effect of interference screw diameter on fixation of soft-tissue grafts in anterior cruciate ligament reconstruction. Arthroscopy 2010;26:1105-10.

7. Siebold R, Dejour D, Zaffagnini S. Anterior Cruciate Ligament Reconstruction. A Practical Surgical Guide. Heidelberg: Springer, 2014.

8. Herrera A, Martinez F, Iglesias D, Cegonino J, Ibarz E, Gracia L. Fixation strength of biocomposite wedge interference screw in ACL reconstruction: effect of screw length and tunnel/ screw ratio. A controlled laboratory study. BMC Musculoskelet Disord 2010; 11:139.
9. Suk N, Heywood CS, Bravman JT, Ieyasa K, Kummer FJ, Meislin RJ. The effect of interference screw diameter on soft tissue graft fixation. Bull NYU Hosp Jt Dis 2006;63:153-5.

10. Morris MW, Williams JL, Thake AJ, Lang Y, Brown JN. Optimal screw diameter for interference fixation in a bone tunnel: a porcine model. Knee Surg Sports Traumatol Arthrosc 2004;12:486-9.

11. Simonian PT, Wickiewicz TL, O’Brien SJ, Dines JS, Schatz JA, Warren RF. Pretibial cyst formation after anterior cruciate ligament surgery with soft tissue autografts. Arthroscopy 1998; 14:215-20.

12. Hunt P, Scheffler SU, Unterhauser FN, Weiler A. A model of soft-tissue graft anterior cruciate ligament reconstruction in sheep. Arch Orthop Trauma Surg 2005;125:238-48.

13. Steiner ME, Hecker AT, Brown CH Jr, Hayes WC. Anterior cruciate ligament graft fixation: comparison of hamstring and patellar tendon grafts. Am J Sports Med 1994;22:240-6.

14. Kurosaka M, Yoshiya S, Andrish JT. A biomechanical comparison of different surgical techniques of graft fixation in anterior cruciate ligament reconstruction. Am J Sports Med 1987; 15:225-9.

15. Gerich TG, Cassim A, Lattermann C, Lobenhoffer HP. Pullout strength of tibial graft fixation in anterior cruciate ligament replacement with a patellar tendon graft: interference screw versus staple fixation in human knees. Knee Surg Sports Traumatol Arthrosc 1997;5:84-8.

16. Bauer LAR, Alberti HAA, Corotti VGP, Franco APGO, Stieven FE, Cunha LAMD. Biomechanical analysis of a double fixation method for tendon graft in porcine tibia using an interference screw plus staple. Rev Bras Ortop 2018;53:564-9.

17. Hill PF, Russell VJ, Salmon LJ, Pinczewski LA. The influence of supplementary tibial fixation on laxity measurements after anterior cruciate ligament reconstruction with hamstring tendons in female patients. Am J Sports Med 2005;33:94-101.

18. Prado M, Martín-Castilla B, Espejo Reina A, Serrano-Fernández JM, Pérez-Blanca A, Ezquerro F. Close-looped graft suturing improves mechanical properties of interference screw fixation in ACL reconstruction. Knee Surg Sports Traumatol Arthrosc 2013; 21:476-84.

19. Teo W, Yeoh C, Wee T. Tibial fixation in anterior cruciate ligament reconstruction. J Orthop Surg (Hong Kong) 2017;25: 2309499017699743.

20. Graf-Alexiou L, Karpyshyn J, Baptiste JJ, Hui C, Sommerfeldt M, Westover L. Biomechanical strength of all-inside ACL reconstruction grafts using side-to-side and backup fixation. Orthop J Sports Med 2021;9:23259671211006521.

21. Bowes J, Mohamed N, Baptiste JJ, Westover L, Hui C, Sommerfeldt M. Biomechanical comparison of graft preparation techniques for all-inside anterior cruciate ligament reconstruction. Orthop J Sports Med 2020;8:2325967120938039.

22. Martini L, Fini M, Giavaresi G, Giardino R. Sheep model in orthopedic research: a literature review. Comp Med 2001;51:2929. 